\title{
Exciton Scattering Approach for Optical Spectra Calculations in Branched Conjugated Macromolecules
}

\author{
Hao Li, ${ }^{1}$ Chao Wu, ${ }^{2}$ Sergey V. Malinin, ${ }^{3}$ Sergei Tretiak, $,{ }^{4,5}, *$ and Vladimir Y. Chernyak ${ }^{3, \dagger}$ \\ ${ }^{1}$ Department of Chemistry, University of Houston, Houston, TX 77204 \\ ${ }^{2}$ Electronic Structure Lab, Center of Microscopic Theory and Simulation, \\ Frontier Institute of Science and Technology, Xian Jiaotong University, Xian, 710054, China \\ ${ }^{3}$ Department of Chemistry, Wayne State University, 5101 Cass Avenue, Detroit, MI 48202 \\ ${ }^{4}$ Theoretical Division and Center for Nonlinear Studies, \\ Los Alamos National Laboratory, Los Alamos, NM 87545 \\ ${ }^{5}$ Center for Integrated Nanotechnologies, Los Alamos National Laboratory, Los Alamos, NM 87545
}

\begin{abstract}
The exciton scattering (ES) technique is a multiscale approach based on the concept of a particle in a box and developed for efficient calculations of excited-state electronic structure and optical spectra in low-dimensional conjugated macromolecules. Within the ES method, electronic excitations in molecular structure are attributed to standing waves representing quantum quasi-particles (excitons), which reside on the graph whose edges and nodes stand for the molecular linear segments and vertices, respectively. Exciton propagation on the linear segments is characterized by the exciton dispersion, whereas exciton scattering at the branching centers is determined by the energy-dependent scattering matrices. Using these ES energetic parameters, the excitation energies are then found by solving a set of generalized "particle in a box" problems on the graph that represents the molecule. Similarly, unique energy-dependent ES dipolar parameters permit calculations of the corresponding oscillator strengths, thus, completing optical spectra modeling. Both the energetic and dipolar parameters can be extracted from quantum-chemical computations in small molecular fragments and tabulated in the ES library for further applications. Subsequently, spectroscopic modeling for any macrostructure within a considered molecular family could be performed with negligible numerical effort. We demonstrate the ES method application to molecular families of branched conjugated phenylacetylenes and ladder poly-para-phenylenes, as well as structures with electron donor and acceptor chemical substituents. Time-dependent density functional theory (TD-DFT) is used as a reference model for electronic structure. The ES calculations accurately reproduce the optical spectra compared to the reference quantum chemistry results, and make possible to predict spectra of complex macromolecules, where conventional electronic structure calculations are unfeasible.
\end{abstract}

\section{INTRODUCTION: THE ES CONCEPT}

Organic conjugated materials have attracted considerable attention for their technological advantages, interesting photophysical and photochemical properties, low cost, light weight, flexibility, and convenience of solutionbased processing techniques [1-8]. The remarkable electronic and optical properties of conjugated molecules are attributed to delocalized $\pi$-electron systems that reside on the quasi-one-dimensional molecular backbones, which can be constructed and tuned synthetically [913]. Therefore, synthetic design of conjugated molecular structures targeting specific optoelectronic properties, requires theoretical understanding and numerical modeling of their electronic excitations. However, the excited state electronic structure of conjugated molecules is complex due to their low dimensionality, strong electronic correlations and significant electron-phonon coupling [14-17]. Only a few quantum-chemical methodologies that are capable of adequate accounting for electron exchange and correlations, can be applied to serve the above purpose [18-20]. Alas, even numerically efficient

*Electronic address: serg@lanl.gov

${ }^{\dagger}$ Electronic address: chernyak@chem. wayne.edu methods that satisfy the above requirements (such as time-dependent density functional theory, TD-DFT [21]), are prohibitively expensive for the excited states computations of large systems, due to the unfavorable scaling of the numerical cost $\left[\mathcal{O}\left(N^{2}\right)-\mathcal{O}\left(N^{5}\right), N\right.$ with being the number of electron orbitals] [22-24]. On the other hand, even large conjugated structures consist of a limited number of elementary molecular blocks $[2,11]$. This opens an opportunity to apply a multiscale concept for efficient description of electronic excitations in a macromolecule by first characterizing the excited states of each building block followed by expressing the electronic properties of the entire structure in terms of the building block data. The exciton scattering (ES) approach $[16,25]$ accomplishes this goal by introducing a rigorous algorithm based on quasi-particle description of electronic excitations.

Indeed, previous studies in the 1990s interpreted excited electronic states in quasi-one-dimensional organic semiconductors as bound electron-hole pairs (excitons) with an emphasis on the relative motion of electrons and holes $[26,27]$. Consequently, a simple and intuitive picture of optical responses emerged from a real-space analysis of the single-electron transition density matrices defined as

$$
\left(\xi_{\nu}\right)_{n m}=\left\langle\nu\left|c_{n}^{\dagger} c_{m}\right| g\right\rangle .
$$


Here, $|g\rangle(|\nu\rangle)$ denotes the ground (excited) state manyelectron wavefunction, $c_{n}^{\dagger}\left(c_{m}\right)$ is the Fermi creation (annihilation) operator of the $n$-th $(m$-th) atomic orbital. Subsequent investigations on electron energy loss spectroscopy (EELS) in linear conjugated oligomers [28, 29] revealed an importance of the "center-of-mass" exciton motion related to the diagonal direction in the transition density matrix (Eq. 1), in contrast to the off-diagonal pattern characterizing the exciton type.

The concept of the ES method first formulated in Ref. [16] attributes a branched conjugated macromolecule (e.g., Fig. 1a) to a graph (Fig. 1b), whose edges and nodes represent the linear segments (composed of the repeat units) and molecular vertices (Fig. 1c), respectively. Exciton motions on such graph form plane waves that scatter at branching centers, joints, and molecular termini. Here both electron and hole move together, and a typical distance between these particles (exciton size) $l_{e}$ can be quantified as the off-diagonal extent of the transition density matrices (see contour plots in Fig. 1e). In the simplest case of a perfect infinite linear chain, the exciton quasimomentum $k$ is a well-defined quantum number that reflects translational symmetry. Therefore, all electronic excitations can be characterized by the dispersion relation $\omega(k)$, which connects the exciton frequency (energy) $\omega$ to its momentum. In finite oligomers, electronic states become discrete, and each state features a characteristic standing wave structure in the diagonal direction of the the transition density matrix. For example, the contour plots of the latter in Fig. 1e correspond to the lowest five excitations in a linear molecule P10 (Fig. 1d). This standing wave pattern is observed in all conjugated macrostructures (e.g., Fig. 1a), where the exciton size $l_{e}$ is small compared to the typical linear segment length $L$. Therefore, the proposed quasiparticle representation, which is asymptotically exact in the long segment $L \gg l_{e}$ limit, is expected to be adequate. Exciton scattering at branching centers and molecular termini are further described using frequency-dependent $n \times n$ scattering matrices $\Gamma^{(n)}(\omega), n$ denoting the vertex degree. These quantities can be viewed as generalized boundary conditions that represent the relevant microscopic properties of the building blocks, rather than introduced purely phenomenologically $[30,31]$. Matrices $\Gamma^{(n)}(\omega)$ and the exciton dispersion $\omega(k)$ fully determine the exciton motion. Consequently, finding the excited states in a molecule is equivalent to solving a generalized spectral problem for a particle in a quasi-one-dimensional box, which is a $2 N \times 2 N$ linear system for the plane wave amplitudes (two per segment), $N$ being the number of linear segments in the molecule under consideration [25, 30, 31].

All ES parameters described above can be obtained using traditional "reference" quantum-chemical excited states calculations in relatively simple molecular fragments associated with the building blocks of the original superstructure [25, 30-33]. Previously, applications of the ES approach has been made to a variety of molecular systems. For example, the exciton spectra $k(\omega)$ have been extracted for phenylacetylene (PA) oligomers and donor-acceptor molecules [25, 31, 32, 34]. Furthermore, the scattering matrices of symmetric double, triple, and quadruple joints have been retrieved by utilizing their geometrical symmetries [33]. To model molecular optical spectra, a technique for the transition dipole calculations within the ES framework has been developed [34-36]. It requires the dipolar (optical) ES parameters, transition charge $q(\omega)$ and transition dipole $\mathbf{d}(\omega)$, which can also be retrieved from quantum-chemical computations in molecules (fragments) of moderate size. As reference quantum-chemical methodologies, we have been using both semiempirical and $a b$ initio techniques. The former applications [32-35] were based on the Collective Electronic Oscillator (CEO) method, which uses the Time-Dependent Hartree-Fock (TDHF) approach combined with the semiempirical Hamiltonians [17, 27]. The latter applications $[25,36]$ relied on TD-DFT electronic structure approach [21, 37]. Overall, all these studies have shown excellent agreement for the excitation energies and optical spectra by comparing the results obtained using the ES method with the full reference quantum-chemical computations. Thus, the ES approach can be readily applied for a broad variety of macromolecular systems as an extension of the currently available quantum-chemical methods for excited state calculations.

In this article we summarize development of the ES theoretical framework and demonstrate applications of this technique to several molecular systems, using modern TD-DFT as a reference electronic structure method for excited state calculations in small molecular fragments. The manuscript is organized as follows: Section II introduces the main ES equations for electronic transition energies and dipole moments. Section III describes the ES parameters extraction techniques illustrated by calculations in representative molecular systems. After tabulating the scattering matrices of molecular building blocks, we demonstrate application of the ES method to computation of vertical absorption spectra in large conjugated macromolecules.

\section{THE ES FORMALISM}

Commonly used quantum-chemical calculations of molecular excited states [37] provide electronic transition energies $\omega_{\nu}$ and the corresponding transition density matrices $\xi_{\nu}$ (Eq. 1). The transition dipole moments $\boldsymbol{\mu}_{\nu}$ between the ground and excited states are further evaluated using the matrix elements of the dipole operator $\hat{\boldsymbol{\mu}}(\mathbf{r})$ in the basis set of atomic orbitals $\left(\hat{\boldsymbol{\mu}}_{n m}=\langle n|\hat{\boldsymbol{\mu}}(\mathbf{r})| m\rangle\right)$. For example, in the orthogonal basis set $\boldsymbol{\mu}_{\nu}$ is given by

$$
\boldsymbol{\mu}_{\nu}=\operatorname{Tr}\left(\hat{\boldsymbol{\mu}} \xi_{\nu}\right) \text {. }
$$

Both $\omega_{\nu}$ (defining positions of resonances) and $\boldsymbol{\mu}_{\nu}$ (defining intensities of optical transitions) are necessary to calculate molecular optical spectra. Here we formulate a closed set of the ES equations relating $\omega_{\nu}$ and $\boldsymbol{\mu}_{\nu}$ to the 
scattering parameters: dispersion $\omega(k)$, matrices $\Gamma^{(n)}(\omega)$, transition charges $q(\omega)$ and transition dipoles $\mathbf{d}(\omega)$.

A branched conjugated molecule (e.g., Fig. 1a) can be viewed as a graph (Fig. 1b) composed of linear segments consisting of identical repeat units [38-40]. The repeat unit and molecular vertices, including branching centers and termini, are referred to as the molecular building blocks that form the molecular backbones. Figure 1c shows typical building blocks of the PA molecules. Since the number of building block types for a given molecular family is usually limited, it is possible to characterize each building block by a tabulated ES parameter library with a limited effort. In an infinite, perfectly straight segment, exciton dispersion $\omega(k)$ fully characterizes the exciton band, being a property of a repeat unit. In a finite linear chain, exciton motion can be represented by two plane waves that propagate in opposite directions. Their quasimomenta have the same absolute value $\omega(k)=\omega(-k)$ due to the time reversal symmetry (in the absence of magnetic fields). Hence, the distribution of an electronic excitation is described by the exciton wave function consisting of standing waves on each linear segment $\alpha$

$$
\psi_{\alpha}\left(x_{\alpha}\right)=a_{\alpha} \exp \left(i k x_{\alpha}\right)+b_{\alpha} \exp \left(-i k x_{\alpha}\right),
$$

where $x_{\alpha}$ denotes integer coordinates of repeat units on a segment $\alpha$ with length $l_{\alpha}$. The exciton quasimomentum $k$ resides in a $1 \mathrm{D}$ Brillouin zone $0 \leq k \leq 2 \pi$, with the points $k$ and $k+2 \pi$ naturally identified. The standing-wave nature of electronic excitations can be directly observed from quantum-chemical computations [16, 27]. For instance, Fig. 1e displays low-energy electronic excitations from the ground state for an example of linear molecule. Here, the contour plots of the absolute values of the transition density matrices are prepared according to the index of atoms along the molecular chain. Apparent standing-wave profile can be readily recognized from the diagonal amplitudes, composed from different contributions of molecular repeat units, with respect to the entire length of the oligomer. The deviation of the exciton wave function from the standing-wave profile is considered as a localized effect at the vertex. We adopt the following normalization condition for the exciton wave functions $\sum_{\alpha} \sum_{x_{\alpha}=1}^{l_{\alpha}} \psi_{\alpha}\left(x_{\alpha}\right) \psi_{\alpha}^{*}\left(x_{\alpha}\right)=1$.

We further introduce a vector wave function

$$
\boldsymbol{\psi}_{\alpha}(x)=\boldsymbol{\sigma}_{\alpha}(x) \psi_{\alpha}\left(x ; \boldsymbol{\sigma}_{\alpha}(x)\right),
$$

where $\boldsymbol{\sigma}_{\alpha}(x)$ is a unit vector in the repeat unit $x$ axis direction, and the scalar function $\psi_{\alpha}\left(x ; \boldsymbol{\sigma}_{\alpha}(x)\right)$ is the projection of the exciton wave function on that axis. In a perfectly straight segment, $\sigma_{\alpha}(x)$ can be defined as a sign factor due to $\sigma_{\alpha}(x)=\sigma_{\alpha}$. Therefore, the sign of the scalar function is altered if the segment orientation is reversed, $\psi_{\alpha}\left(x ;-\boldsymbol{\sigma}_{\alpha}\right)=-\psi_{\alpha}\left(x ; \boldsymbol{\sigma}_{\alpha}\right)$. The representation of Eq. (4) is especially useful for complex structures with slightly bent segments (e.g., loops), where $\boldsymbol{\sigma}_{\alpha}(x)$ varies slowly as a function of $x[30-32,35]$.
The excitation amplitudes can then be found by solving linear equations, which describe exciton propagation along the linear segments and exciton scattering at the molecular vertices. We denote the outgoing $(+)$ and incoming (-) plane waves on segment $\alpha$ at vertex $b$ by $\boldsymbol{\psi}_{\alpha b}^{(+)}$and $\boldsymbol{\psi}_{\alpha b}^{(-)}$, respectively. Exciton propagation along segment $\alpha$ between vertices $b$ and $c$ is described by

$$
\boldsymbol{\psi}_{\alpha b}^{(-)}=\boldsymbol{n}_{b \alpha}\left(\boldsymbol{n}_{c \alpha} \cdot \boldsymbol{\psi}_{\alpha c}^{(+)}\right) \exp \left(i k l_{\alpha}\right),
$$

where $l_{\alpha}$ is the segment $\alpha$ length, $\boldsymbol{n}_{u \alpha}$ stands for the unit vector of the repeat unit in segment $\alpha$ attached to the vertex $u$. There are two such equations for each segment that correspond to its two ends. At molecular vertex $b$ that connects $n$ linear segments, the exciton scattering is described by a frequency-dependent $n \times n$ scattering matrix $\Gamma_{b}(\omega)$, which transforms the incoming to the outgoing waves:

$$
\boldsymbol{\psi}_{\alpha b}^{(+)}=\boldsymbol{n}_{b \alpha} \sum_{\beta \ni b} \Gamma_{b, \alpha \beta}\left(\omega ; \boldsymbol{n}_{b}\right)\left(\boldsymbol{n}_{b \beta} \cdot \boldsymbol{\psi}_{\beta b}^{(-)}\right),
$$

$\boldsymbol{n}_{b}$ denoting a set of unit vectors, associated with the branches attached to vertex $b$. The off-diagonal elements of $\Gamma_{b}$ that determine the transmission effects at a joint, are orientation-dependent due to the vector nature of the incoming and outgoing waves. In contrast, the diagonal elements of $\Gamma_{b}$ related to reflections, are invariant with respect to the orientation choice.

The ES equations (5) and (6) constitute a frequencydependent homogeneous system of linear equations for the plane wave amplitudes $\boldsymbol{\psi}_{\alpha b}^{( \pm)}$, with the number of equations matching the number of unknown variables $[16,30]$ that can be referred to as a generalized spectral problem. Therefore, given the exciton dispersion $\omega(k)$ and the scattering matrices $\Gamma(\omega)$ for all vertices, excitation energies and exciton wave functions (up to a normalization factor) can be identified by solving the generalized spectral problem [Eqs. (5) and (6)] [30-32].

To perform the transition dipole calculations we further introduce the transition charge and transition dipole parameters of the building blocks. An exciton wavefunction $\psi(x)=a \exp (i k x)+b \exp (-i k x)$ is associated with the transition dipole distribution and its dual $\tilde{\psi}(x)=$ $a \exp (i k x)-b \exp (-i k x)$ represents the transition charge distribution. $\psi$ and $\tilde{\psi}$ are related by

$$
\tilde{\psi}(x)=\frac{1}{k} \operatorname{div} \psi(x) \equiv \frac{1}{k} \frac{d(\boldsymbol{\sigma}(x) \cdot \psi[x ; \boldsymbol{\sigma}(x)])}{d x},
$$

i.e., the "charge" wavefunction can be viewed as the divergence of the "dipole" counterpart [35].

Far from the scattering centers, the total transition dipole has contributions from the transition dipoles and charges of the repeat units, proportional to the amplitudes of the exciton wave function and its dual counterpart, respectively. This allows to introduce the frequency-dependent dipole and charge parameters of the repeat unit, that, being weighted with the proper exciton wave function, yield the repeat unit contributions 
to the total transition dipole. The deviations of transition charges and dipoles from the standing-wave form in the repeat units, located in the close proximity of the scattering centers, can be further "absorbed" into the vertex contributions. For any scattering center we can choose the amplitudes of either incoming or outgoing waves in the wave function and its dual to characterize the weights, associated with the vertex, since the outgoing and incoming amplitudes are related to each other via Eqs. (6) and (7). In the case of incoming waves, the total transition dipole can be expressed as the sum of contributions of all repeat units (the first term) and all vertices (the second term):

$$
\begin{aligned}
\boldsymbol{\mu}= & \sum_{\alpha} \sum_{x_{\alpha}=1}^{l_{\alpha}}\left(q(\omega) \boldsymbol{r}_{x_{\alpha}}^{(\alpha)} \tilde{\psi}_{\alpha}\left(x_{\alpha}\right)+d(\omega) \boldsymbol{\psi}_{\alpha}\left(x_{\alpha}\right)\right) \\
& +\sum_{b} \sum_{\alpha \ni b}\left(q_{b \alpha}(\omega) \boldsymbol{r}_{b} \tilde{\psi}_{\alpha b}^{(-)}+d_{b \alpha}(\omega) \boldsymbol{\psi}_{\alpha b}^{(-)}\right)
\end{aligned}
$$

where $q(\omega)$ and $d(\omega) \quad\left(q_{b \alpha}(\omega)\right.$ and $\left.d_{b \alpha}(\omega)\right)$ are the frequency-dependent transition charge and dipole parameters of the molecular repeat unit (vertices), respectively. The dipole parameters are rank 2 tensors since they provide linear transformations between the two vectors, the exciton wave function and the transition dipole. $\boldsymbol{r}_{x_{\alpha}}^{(\alpha)}$ and $\boldsymbol{r}_{b}$ denote the positions of the repeat unit $x_{\alpha}$ in segment $\alpha$ and vertex $b$, respectively. Thus, Eq. (8) provides an expression for the transition dipole between the ground and excited electronic state, the latter described by its exciton wavefunction and its dual counterpart.

\section{APPLICATION EXAMPLES}

The first step in applying the ES approach to an arbitrary molecular structure is to separate the latter into building blocks such as linear segments and scattering centers (e.g., branching units, joints, termini, kinks, etc.). The definition of building blocks in not unique and can be further fine-tuned depending on the accuracy of the ES results compared to the reference quantum-chemical simulations. The next step is selection of the reference quantum-chemical approach (see our discussion in the next sub-section) and performing electronic structure simulations of the subset of the building blocks aiming to tabulate the ES parameters as illustrated on the numerous examples in this section. Formally, this is the most numerically costly step, yet it is enormously easier compared to the complete quantum-chemical supramolecular calculation in the entire structure. The ES approach itself boils down to solving Eqs. (5) and (6) that results in obtaining transition frequencies and exciton wavefunctions [25, 32, 34]. The latter, being substituted into Eq. (8) allows the transition dipoles do be computed $[35,36]$. The above procedure is extremely efficient, provided the building blocks are already characterized by previously tabulated ES parameters. The aforementioned step can be repeated as many times as necessary, for example, to explore excited states in different molecular structures composed from the same building blocks. Here we illustrate how these parameters can be extracted from quantum-chemical computations in molecular fragments of moderate size. Absorption spectra of several molecules are further calculated using the ES approach and compared to their counterparts obtained through direct reference quantum-chemical computations.

\section{A. Reference electronic structure theory}

Any size-consistent quantum-chemical method, capable of describing the bound nature of excitonic states, can be used as a reference electronic structure theory for the ES approach [18-20]. Previously, we relied on both semiempirical CEO and $a b$ initio TD-DFT approaches as reference quantum-chemical techniques. Notably, only hybrid DFT kernels or modern long-range corrected functionals can adequately describe exciton binding energies [19]. Here, a hybrid functional subjected to Coulomb-attenuating method (CAM-B3LYP) [41, 42] and 6-31G basis set were chosen for all TD-DFT computations, adopted as a reference quantum chemistry approach for excited-state description. The molecular ground state geometries have been optimized at the same CAM-B3LYP/6-31G level. All simulations have been performed with the Gaussian 09 package [43].

To exemplify the ES modeling, we use molecules based on phenylacetylene (PA) and ladder poly-para-phenylenes (PPP) backbones (see Fig. 2 inset). Excited states in the selected molecular fragments (as detailed below) have been computed with the reference TD-DFT approach, resulting in the vertical excitation energies, oscillator strengths and transition density matrices. In this Account we focus on the lowest energy optically active $\pi$-exciton band (similar analysis can be applied to any other excitonic band of interest) [30-32]. Calculated electronic states belonging to this band have been singled out by inspecting the corresponding transition density matrices. Their excitations energies are sufficient to compute $\omega(k)$ and $\Gamma(\omega)$ scattering sets $[25,31,32]$. To calculate the ES dipole parameters, we recall that, to efficiently separate the dipolar contributions of individual building blocks, it is highly desirable to have the basis functions to be both orthogonal and spatially localized. In contrast, $a b$ initio methods typically rely on non-orthogonal and frequently over-complete atomic orbital (AO) basis set, and the commonly used Löwdin orthogonalization [44] is not adequate due to the loss of the angular momentum symmetries in the original AOs [36]. The Natural Atomic Orbitals (NAO) constructed from the occupancy-weighted symmetric orthogonalization procedure preserve atom-like character of angular-symmetry within the molecule [45]. Not surprisingly, the NAO transformation is found to be well suited for calculating the ES dipolar parameters [36] and is readily available 
from the NBO program [46] built in the major electronic structure codes such as Gaussian 09 [43]. Here, the transition density matrix $\xi_{\nu}$ and dipole matrix $\hat{\boldsymbol{\mu}}$ are obtained using the transformation matrix $\boldsymbol{T}$ from $\mathrm{AO}$ to NAO space as

$$
\xi_{\nu}^{(\mathrm{NAO})}=\boldsymbol{T} \xi_{\nu}^{(\mathrm{AO})} \boldsymbol{T}^{\dagger}, \hat{\boldsymbol{\mu}}^{(\mathrm{NAO})}=\boldsymbol{T} \hat{\boldsymbol{\mu}}^{(\mathrm{AO})} \boldsymbol{T}^{\dagger} .
$$

Consequently, all dipolar parameters are calculated using the NAO representation. For example, the transition charge of a building block $N$ is given by $q_{\nu}^{N}=\operatorname{Tr}\left(\xi_{\nu}^{N}\right)$ instead of the ambiguous Mulliken charge in the AO space.

\section{B. ES modeling of simple linear chains}

Symmetries possessed by quantum systems simplify the extraction of the ES parameters. Time-reversal symmetry leads to symmetric exciton dispersion $\omega(k)=$ $\omega(-k)$ and certain symmetry in matrices $\Gamma(\omega)$. Unitarity, associated with the probability conservation in evolution of quantum systems, is another universal symmetry of the scattering matrices. Finite size linear PA and PPP chains terminated by neutral (hydrogen atom), donor $\left(-\mathrm{NH}_{2}\right)$ or acceptor $\left(-\mathrm{NO}_{2}\right)$ group (see insets in Fig. 2) provide simple illustrative examples for the ES theory. Due to the unitarity, a single reflection phase $\phi_{T}(\omega)$ parameterizes the scattering matrix $\Gamma(\omega)=e^{i \phi_{T}(\omega)}$ of a molecular terminus (Fig. 1c(ii)). Therefore, the dispersion $\omega(k)$ and reflection phases $\phi_{T}(\omega)$ of different termini represent a closed set of the ES parameters for these systems. In linear molecules terminated by $A$ and $B$ end groups, the wave amplitudes in the exciton wave function (Eq. (3)) are related by Eq. (6) imposed on the two molecular termini $[25,31]$. These two ES equations have non-trivial solutions only if the following quantization condition is satisfied:

$$
2 k(\omega) L+\phi_{A}(\omega)+\phi_{B}(\omega)=2 \pi q,
$$

where $L$ is molecular length (the number of repeat units) and $q$ is an integer that labels the excitation, which is related to the number of nodes in the exciton wave function (Fig. 1e). $\phi_{A}$ and $\phi_{B}$ are the reflection phases of the two termini $A$ and $B$, respectively. It is worth mentioning that the ES approach does not depend on the choice of repeat unit because the choice affects the segment length $L$ thence the quasimomentum $k$ in Eq. (10) in a consistent way so that the solutions of the ES equations remain the same.

In the simplest case of linear molecules without chemical substituents, both termini are identical $\phi_{A}=\phi_{B}=$ $\phi_{T}, \phi_{T}$ being the reflection phase of the neutral $-\mathrm{H}$ terminus. Therefore, Eq. (10) is simplified as $k L+\phi_{T}=\pi q$, where two functions $k(\omega)$ and $\phi_{T}(\omega)$ should be evaluated simultaneously. The range of reflection phases $\phi_{T}(\omega)$ is determined by the definition of $q$, since $\phi_{T}(\omega)$ is a periodic function of $2 \pi$. Therefore, to have $\pi<\phi_{T}<2 \pi$ (considering the hard wall reflection phase $\phi_{T}=\pi$ ), the integer $q$ is related to the number of nodes in the exciton wave function, given by $q-2[30,31]$. Accordingly, $q$ can be determined from the transition density matrix (see Fig. 1e). The oligomers suitable for the ES parameter extraction should be longer than the exciton size being 2-3 repeat units for phenylacetylene excitons (Fig. 1e). Indeed, quantum chemistry calculations of 10 linear PA and PPP molecules (Fig. 2a) of different lengths (10-25 repeat units) proved to be sufficient to accurately extract $k(\omega)$ and $\phi_{T}(\omega)$ simultaneously. Namely, using excitation energies and respective transition density matrices, the initial values of $k(\omega)$ and $\phi_{T}(\omega)$ have been derived from two- and four- point numerical extrapolations [31] followed by a piecewise polynomial least-square fit. This procedure produces smooth functions $k(\omega)$ and $\phi_{T}(\omega)$ tabulated for their future use (see Fig. 2).

Substitutions of conjugated oligomers with polar moieties perturb the $\pi$-electron system and possibly break the electron-hole symmetry, thus representing a useful synthetic approach to tune molecular electronic and optical properties. Here the effect of chemical modifications on the optical properties appears via correction of the corresponding ES parameters. Linear oligomers substituted with donor $\left(-\mathrm{NH}_{2}\right)$ or acceptor $\left(-\mathrm{NO}_{2}\right)$ group on one side (see Fig. 2b inset) are the simplest cases allowing to extract the reflection phases $\phi_{X}(\omega)$ for the termini with substituents (i.e., $\phi_{A}=\phi_{T}$ and $\phi_{B}=\phi_{X}$ in Eq. (10)). Using previously retrieved $k(\omega)$ and $\phi_{T}(\omega), \phi_{X}(\omega)$ can be readily tabulated from the excitation energies in such molecules. Different reflection phases of $-\mathrm{H},-\mathrm{NH}_{2}$ and $-\mathrm{NO}_{2}$ termini shown in Fig. 2b signify changes in the corresponding electronic spectra due to substituents [34].

To extract the ES dipole parameters in linear molecules, we first calculate the exciton wave functions and their duals from Eqs. (5), (6), and (7) using the tabulated ES energy parameters. The transition charges $Q(x)$ and dipoles $\boldsymbol{\mu}(x)$ of all repeat units can be calculated from the transition density matrices in the NAO basis (see Section III A). The charge parameter $q(\omega)$ of a repeat unit can be found by comparing the transition charge to the amplitude of the dual wave function $Q(x) / \tilde{\psi}(x)$. Since the transition dipoles of the lowest band excitons are directed along the chain, the dipole tensor of a repeat unit is a scalar $d(\omega)$ determined by the ratio $\mu(x) / \psi(x)$. The ratios $Q(x) / \tilde{\psi}(x)$ and $\mu(x) / \psi(x)$ deviate from the constants within several repeat units from the termini, hence the latter should be excluded from calculations [35]. Obtained using the above approach functions $q(\omega)$ and $d(\omega)$ (see Fig. 3a) fully characterize the transition dipoles of the PA backbone.

The transition charge and dipole parameters of molecular vertices have internal contributions from all atoms that belong to the vertex (which can be evaluated from the respective transition density matrices). In addition, mentioned above deviations from the standing-wave form in the nearby repeat units (referred to as the external contributions) should be taken into account as well. 
Namely, the deviations of transition charge and dipole on the repeat unit $x$ can be described as

$$
\begin{aligned}
\Delta Q(x) & =Q(x)-q(\omega) \tilde{\psi}_{\alpha}(x), \\
\Delta \boldsymbol{\mu}(x) & =\boldsymbol{\mu}(x)-d(\omega) \boldsymbol{\psi}_{\alpha}(x) .
\end{aligned}
$$

To accurately evaluate these external contributions, it is sufficient to sum within three repeat units of a given scattering scattering center in the PA systems [35]. For molecular terminus, a single parameter is sufficient to represent the dipole tensor. Therefore, we can introduce the real-valued parameters $q^{(T)}(\omega)$ and $d^{(T)}(\omega)$, by comparing the effective transition dipole and charge of the end group to the exciton wave function and its dual, respectively. Following this idea, the ES dipole parameters of $-\mathrm{H},-\mathrm{NH}_{2}$ and $-\mathrm{NO}_{2}$ termini have been extracted, fitted by piecewise polynomials and plotted in Fig. 3b,c. As in the case of reflection phase $\phi(\omega)$, modification of dipole parameters for substituents indicates different optical transition intensities in the functionalized oligomers [34].

To this point, the optical properties of the lowest band $\pi$-excitons for PA backbone and three different end groups have been characterized by the tabulated ES energy and dipole parameters, which allows to calculate the absorption spectrum of an arbitrary linear PA molecule terminated by any considered substituent. These calculations are practically instantaneous $[25,32,35,36]$. For example, Figure 4 shows comparison of the optical spectra calculated with the ES approach (completely bypassing the electronic structure numerics) and direct quantum-chemical modeling for two different oligomers substituted at the both ends. The transition energy discrepancies between the two methods are less than $2 \mathrm{meV}$, whereas the relative differences of transition dipoles of optically-allowed states are within $3 \%$. This exemplifies efficiency and accuracy of the ES modeling.

\section{Applications of the ES approach to complex branched molecules}

To apply the ES approach to complex molecular structures and networks, scattering centers of higher degree should be considered. We further characterize symmetric $V$ (meta- and ortho-) and triple $Y$ joints, which structures are shown in Fig. 1c. In this case, the joint's scattering matrix $\Gamma(\omega)$ can be diagonalized using irreducible representations of the vertex symmetry group $G$ : $\Gamma=U D U^{\dagger}$, where $D$ is the diagonal matrix with elements in a form $e^{i \phi}[30,31,33]$. Therefore, $\Gamma$ is parameterized by the scattering phases $\phi(\omega)$.

$\Gamma_{V}(\omega)$ of the symmetric $V$ joint is a $2 \times 2$ matrix with the elements $r(\omega)$ and $t(\omega)$, being the complex reflection and transmission amplitudes [25]. The symmetry group of a $V$ joint is $C_{2}$ with two irreducible representations. Therefore, $\Gamma_{V}$ can be parameterized by two real phases $\phi_{0}$ and $\phi_{1}$ corresponding to the symmetric and antisym- metric configurations, denoted by subscripts 0 and 1 , respectively. The scattering matrix can be diagonalized by

$$
\begin{aligned}
& \Gamma_{V}=U D U^{\dagger}=\left(\begin{array}{ll}
r & t \\
t & r
\end{array}\right), \\
& U=\frac{1}{\sqrt{2}}\left(\begin{array}{cc}
1 & 1 \\
1 & -1
\end{array}\right), D=\left(\begin{array}{cc}
e^{i \phi_{0}} & 0 \\
0 & e^{i \phi_{1}}
\end{array}\right) .
\end{aligned}
$$

Consequently, $r(\omega)$ and $t(\omega)$ are related to two real energy-dependent phases $\phi_{0}(\omega)$ and $\phi_{1}(\omega)$ by

$$
r=\frac{1}{2}\left(e^{i \phi_{0}}+e^{i \phi_{1}}\right), \quad t=\frac{1}{2}\left(e^{i \phi_{0}}-e^{i \phi_{1}}\right) .
$$

The symmetry group of a $Y$ joint is $D_{3}$ with six elements including the rotations by $\pm 2 \pi / 3$ and three reflections. Similarly, according to its two irreducible representations: the identity operation has zero angular momentum $m=0$, and rotations result in symmetric and antisymmetric reflections with $m= \pm 1$ [33]. Diagonalization of $\Gamma_{Y}$ can be performed as

$$
\begin{aligned}
\Gamma_{Y} & =U D U^{\dagger} \\
U & =\frac{1}{\sqrt{3}}\left(\begin{array}{ccc}
1 & 1 & 1 \\
1 & \exp (2 \pi i / 3) & \exp (-2 \pi i / 3) \\
1 & \exp (-2 \pi i / 3) & \exp (2 \pi i / 3)
\end{array}\right) \\
D & =\left(\begin{array}{ccc}
\exp \left(i \phi_{S}\right) & 0 & 0 \\
0 & \exp \left(i \phi_{P}\right) & 0 \\
0 & 0 & \exp \left(i \phi_{P}\right)
\end{array}\right)
\end{aligned}
$$

Following the above scheme, $\Gamma_{Y}$ are parameterized by two real energy-dependent phases $\phi_{S}(\omega)$ and $\phi_{P}(\omega)$, associated with the irreducible representations.

The above derivation shows that each scattering phase in symmetric joints is equivalent to the reflection phase of a terminus that represents the joint for transition density matrix of the corresponding symmetry. These phases can be extracted from the quantum-chemical results that possess the required symmetries using Eq. (10). Namely, we calculated symmetric PA fragments of $V$ and $Y$ types that have identical arm length $\mathrm{L}$ (6 repeat units) in each molecule, to preserve the joint symmetry. Using these electronic structure results for the lowest excitonic band and Eq. (10), the scattering phases of meta- $\left(\phi_{0}^{M}(\omega)\right.$ and $\left.\phi_{1}^{M}(\omega)\right)$, ortho- $\left(\phi_{0}^{O}(\omega)\right.$ and $\left.\phi_{1}^{O}(\omega)\right)$ and $Y\left(\phi_{S}(\omega)\right.$ and $\left.\phi_{P}(\omega)\right)$ joints have been further tabulated. Figures $5 \mathrm{a}$ and 6 a display the resulting functions after polynomial fits.

The number of the relevant ES dipole parameters describing a molecular joint is related to the symmetry as well. The transition dipole parameters of planar joints always appear in pairs (projections on two principle axes $x$ and $y$, see Fig. 5a and Fig. 6b inset). The parameter extraction for a molecular vertex is similar to that for the terminus [33, 35]. Real-valued parameters are retrieved with respect to the standing waves instead of plane waves and can be related to the parameters in Eq. (8). The external contributions should be carefully evaluated. For 
example, in $V$ and $Y$ joints, the contribution from the region marked with "-" (due to the repeat unit overlap) in the phenyl ring of the linkage should be subtracted from the one from the region marked with "+" [as shown in Fig. 5a and Fig. 6b inset] [33, 35].

The ES dipole parameters for the $V$ joints are transition charge $q$ and two real dipole parameters $d_{x}$ and $d_{y}$. Here the symmetry of electronic excitations is defined as the symmetry of the $x$ projection of the transition dipole distribution. Therefore, $q$ can only be extracted from the data on the antisymmetric modes, since the symmetry of the exciton dual wave function is opposite to that of the exciton wave function [35]. Thereby, the charge parameters of the meta- $\left(q^{M}(\omega)\right)$ and ortho- $\left(q^{O}(\omega)\right)$ joints have been calculated by comparing the transition charge to the sum of the amplitudes of the dual wave functions on both sides of the joint, using antisymmetric excitations. Furthermore, the respective dipole parameters $d_{y}^{M}(\omega)$ and $d_{x}^{M}(\omega)\left(d_{x}^{O}(\omega)\right.$ and $\left.d_{y}^{O}(\omega)\right)$ are retrieved using the sum of the values of the wave functions and their duals at both sides of the joint. The results are shown in Fig. 5. The ES dipole parameters for symmetric $Y$ joint are transition charge $q^{Y}(\omega)$ and dipole $d^{Y}(\omega)$ (due to symmetry $d_{x}^{Y}=d_{y}^{Y}=d^{Y}$ ) [33]. These quantities have been extracted using similar to the $V$-joint procedure and the resulting functions are plotted in Fig. 6.

At this juncture, our ES library is rich enough to predict optical spectra in large complicated PA based molecular structures and networks. For example, the absorption spectrum of a dendrimer has been calculated under the ES framework and compared to the quantumchemical results in Fig. 6c. We notice that the ES spectrum accurately reproduces the result of the reference method even in macromolecules containing many short segments, comparable in length to the exciton size.

\section{CONCLUSIONS}

In this Account, we reviewed the formalism of the Exciton Scattering (ES) approach developed for spectroscopic calculations in branched conjugated macromolecules, where the electronic excitations can be viewed as quasiparticles (excitons) that reside on the corresponding quasi-1D molecular graphs [16]. The ES methodology has been illustrated using various phenylacetylene based molecules including structures with donor/acceptors substituents with TD-DFT being the reference quantum chemistry method. Exciton dispersion and scattering matrices of commonly used molecular vertices have been extracted, which allows efficient and numerically inexpensive calculations of the excitation energies and exciton wave functions. The transition charge and dipole parameters of aforementioned molecular building blocks are also characterized. The retrieved ES parameters allow for fast calculations of absorption spectra in molecules that consist of the characterized building blocks, no matter how large or complex they are. Being specific, the ES ap- proach reduces the computer time scale to seconds compared to days or even weeks when conventional quantumchemical methods are used. The results accurately reproduce the outcome of time-consuming supramolecular TD-DFT calculations. This became possible because the ES modeling relies solely on the pre-tabulated library of scattering parameters, completely bypassing quantumchemical computations. The accuracy of the ES simulations can be further improved by refining the ES parameters using high-quality spectroscopic data for small molecules representing building blocks [30,31]. Calculations of the ES parameters for complex asymmetric vertices are possible as well using the respective exciton wave functions (in addition to the excitation energies) [31, 32].

Overall, the ES multiscale approach fills the gap between the need for the accurate characterization of optical spectra in conjugated macrostructures and restrictions of general quantum-chemical methods due to high numerical cost. The ES methodology is thus complementary, rather than alternative, to the commonly used quantum-chemical methods and is likely to substantially extend their capability to compute excited-state electronic structure in much larger systems, including supramolecules, superstructures and molecular networks. Moreover, developing extensive sets of scattering parameters for various molecular building block families brings forward a great potential of the ES approach for realtime computational design of molecular structures with desirable electronic and optical properties.

Finally, proposed quasiparticle scattering idea bridges traditional molecular description (spatially confined wavefunctions, orbitals) with solid-state physics picture (periodic delocalized wavefunctions, quasiparticles), providing a universal framework closely related to the conventional condensed matter quasiparticle description of many excitation processes and dynamics such as excitons, polarons, spins, plasmons, etc. Consequently, the ES methodology can be naturally augmented by adding exciton-electron, exciton-phonon and electronphonon scattering processes occurring at the interfaces, chemical defects and impurities. For example, to incorporate electron-phonon coupling and conformational disorder in 'soft' electronic materials, it is possible to reformulate the scattering equations in terms of an effective tight-binding (lattice) model, which is a Hamiltonian describing the parent scattering problem [47].

The tight-binding models can be build in an efficient way by studying the the topological properties of the scattering matrices, namely the integer-valued topological charge, or equivalently winding number associated with the corresponding scattering matrix [48]. The topological charge provides useful information on how many cites in the related tight-binding model are needed to adequately describe a scattering center. Studying analytical (geometrical) properties of the scattering matrices, namely the analytical continuations of $\Gamma(k)$ with $k$ being the quasimomentum, from the Brillouin zone, represented by a unit circle in the complex plane (or 
its compactified version, known as the projective space $\mathbb{C} P^{1}$ ) provide useful microscopic insights into the chemical properties of molecular substituents [49], connected to polymer backbones. The positions of poles (or zeros) of $\Gamma(k)$ in $\mathbb{C} P^{1}$ describe the energies and spectral widths of the bound and resonant states that occur in a conjugated molecule due to the presence of the substituent, with the above energies sometimes being very different form the energetics of an uncoupled fragment. Treating a localized molecular geometry distortion, e.g., a bond-alternation stretch or an around-a-bond rotation as a scattering center for electronic excitations (excitons), allows the dependence of the tight-binding model parameters (on-site energies and hopping constants) to be extracted from quantum chemistry computations [50], which leads eventually to an exciton-phonon Hamiltonian. This allows effects of incoherent energy transfer in a branched conjugated molecule to be treated at the computational cost of a Frenkel-exciton system counterpart.

So far we have been considering polymer backbones with the repeat units that can be more or less adequately described by a single site in the respective tightbinding model. In the case of multiple sites in a repeat unit, or/and longer range hopping (e.g., next to nearestneighbor) analytical properties of the scattering matrices, as well as exciton spectra show more complexity. They can be still efficiently studied, and, according to our preliminary studies (the results will be published elsewhere), the Scattering matrices can be still represented as meromorphic functions on higher Riemann surfaces, or using more mathematical language on algebraic (complexanalytical) curves of higher genus $g$.

\section{Acknowledgments}

This material is based upon work supported by the National Science Foundation under Grant No. CHE1111350. We acknowledge support of Directed Research and Development Funds, Center for Integrated Nanotechnology and Center for Nonlinear Studies at Los Alamos National Laboratory (LANL). LANL is operated by Los Alamos National Security, LLC, for the National Nuclear Security Administration of the U.S. Department of Energy under contract DE-AC52-06NA25396.
[1] Heeger, A. J. Semiconducting polymers: the third generation. Chem. Soc. Rev. 2010, 39, 2354-2371.

[2] Gust, D., Moore, T. A., and Moore, A. L. Mimicking photosynthetic solar energy transduction. Acc. Chem. Res. 2001, 34, 40-48.

[3] Sirringhaus, H., Kawase, T., Friend, R. H., Shimoda, T., Inbasekaran, M., Wu, W., and Woo, E. P. High-resolution inkjet printing of all-polymer transistor circuits. Science 2000, 290, 2123-2126.

[4] Forrest, S. R. The path to ubiquitous and low-cost organic electronic appliances on plastic. Nature 2004, 428, $911-918$.

[5] Murphy, A., and Frechet, J. Organic Semiconducting Oligomers for Use in Thin Film Transistors. Chem. Rev. 2007, 107, 1066-1096.

[6] Barbara, P. F., Gesquiere, A. J., Park, S. J., and Lee, Y. J. Single-molecule spectroscopy of conjugated polymers. Acc. Chem. Res. 2005, 38, 602-610.

[7] Wang, D. L., Gong, X., Heeger, P. S., Rininsland, F., Bazan, G. C., and Heeger, A. J. Biosensors from conjugated polyelectrolyte complexes. Proc. Nat. Acad. Sci. USA 2002, 99, 49-53.

[8] Gunes, S., Neugebauer, H., and Sariciftci, N. Conjugated Polymer-Based Organic Solar Cells. Chem. Rev. 2007, 10\%, 1324-1338.

[9] Bolinger, J. C., Traub, M. C., Brazard, J., Adachi, T., Barbara, P. F., and Bout, D. A. V. Conformation and Energy Transfer in Single Conjugated Polymers. Acc. Chem. Res. 2012, 45, 1992-2001.

[10] Goodson, T. G. Time-Resolved Spectroscopy Of Organic Dendrimers And Branched Chromophores. Ann. Rev. Phys. Chem. 2005, 56, $581-603$.

[11] Goodson, T. G. Optical excitations in organic dendrimers investigated by time-resolved and nonlinear optical spec- troscopy. Acct. Chem. Res. 2005, 38, $99-107$.

[12] Bunz, U. H. F. Poly(arylene etynylene)s: From Synthesis to Application. Adv. Polym. Sci. 2005, 177, 1-52.

[13] Lo, S.-C., and Burn, P. Development of Dendrimers: Macromolecules for Use in Organic Light-Emitting Diodes and Solar Cells. Chem. Rev. 2007, 10\%, 10971116.

[14] Collini, E., and Scholes, G. D. Coherent Intrachain Energy Migration in a Conjugated Polymer at Room Temperature. Science 2009, 323, 369-373.

[15] Scholes, G. D., and Rumbles, G. Excitons in nanoscale systems. Nature Mat. 2006, 5, 683 - 696.

[16] Wu, C., Malinin, S. V., Tretiak, S., and Chernyak, V. Y. Exciton scattering and localization in branched dendrimeric structures. Nature Phys. 2006, 2, $631-635$.

[17] Schulz, M., Tretiak, S., Chernyak, V. Y., and Mukamel, S. Size Scaling of Third-Order Off-Resonant Polarizabilities. Electronic Coherence in Organic Oligomers. J. Am. Chem. Soc. 2000, 122, 452-459.

[18] Ruini, A., Caldas, M. J., Bussi, G., and Molinari, E. Solid state effects on exciton states and optical properties of PPV. Phys. Rev. Lett. 2002, 88, $206403-4$.

[19] Igumenshchev, K. I., Tretiak, S., and Chernyak, V. Y. Excitonic effects in a time-dependent density functional theory. J. Chem. Phys. 2007, 127, $1-10$.

[20] Rohlfing, M., and Louie, S. G. Optical excitations in conjugated polymers. Phys. Rev. Lett. 1999, 82, 1959 - 62.

[21] Tretiak, S., and Chernyak, V. Resonant Nonlinear Polarizabilities in the Time-Dependent Density Functional (TDDFT) theory. J. Chem. Phys. 2003, 119, 8809 8823.

[22] Caricato, M., Trucks, G. W., and Frisch, M. J. A Comparison of Three Variants of the Generalized Davidson Algorithm for the Partial Diagonalization of Large Non- 
Hermitian Matrices. J. Chem. Theory Comput. 2010, 6, 1966-1970.

[23] Tretiak, S., Isborn, C. M., Niklasson, A. M. N., and Challacombe, M. Representation Independent Algorithms for Molecular Response Calculations in Time-Dependent Self-Consistent Field Theories. J. Chem. Phys. 2009, $130,054111$.

[24] Chernyak, V. Y., Schulz, M., Mukamel, S., Tretiak, S., and Tsiper, E. V. Krylov-Apace Algorithms for TimeDependent HartreeFock and Density Functional Computations. J. Chem. Phys. 2000, 113, 36-43.

[25] Wu, C., Malinin, S. V., Tretiak, S., and Chernyak, V. Y. Multiscale modeling of electronic excitations in branched conjugated molecules using an exciton scattering approach. Phys. Rev. Lett. 2008, 100, 057405.

[26] Mukamel, S., Tretiak, S., Wagersreiter, T., and Chernyak, V. Electronic coherence and collective optical excitations of conjugated molecules. Science 1997, 277, 781-787.

[27] Tretiak, S., and Mukamel, S. Density matrix analysis and simulation of electronic excitations in conjugated and aggregated molecules. Chem. Rev. 2002, 102, 3171-3212.

[28] Chernyak, V., Volkov, S. N., and Mukamel, S. Exciton coherence and electron energy loss spectroscopy of conjugated molecules. Phys. Rev. Lett. 2001, 86, 995-998.

[29] Chernyak, V., Volkov, S. N., and Mukamel, S. Electronic structure-factor, density matrices, and electron energy loss spectroscopy of conjugated oligomers. J. Phys. Chem. A 2001, 105, 1988-2004.

[30] Wu, C., Malinin, S. V., Tretiak, S., and Chernyak, V. Y. Exciton Scattering Approach for Branched Conjugated Molecules and Complexes. I. Formalism. J. Chem. Phys. 2008, 129, 174111.

[31] Wu, C., Malinin, S. V., Tretiak, S., and Chernyak, V. Y. Exciton Scattering Approach for Branched Conjugated Molecules and Complexes. II. Extraction of the Exciton Scattering Parameters from Quantum-Chemical Calculations. J. Chem. Phys. 2008, 129, 174112.

[32] Wu, C., Malinin, S. V., Tretiak, S., and Chernyak, V. Y. Exciton Scattering Approach for Branched Conjugated Molecules and Complexes. III. Applications. J. Chem. Phys. 2008, 129, 174113.

[33] Li, H., Wu, C., Malinin, S. V., Tretiak, S., and Chernyak, V. Y. Exciton Scattering on Symmetric Branching Centers in Conjugated Molecules. J. Phys. Chem. B 2011, 115, 5465-5475.

[34] Li, H., Wu, C., Malinin, S. V., Tretiak, S., and Chernyak, V. Y. Excited States of Donor and Acceptor Substituted Conjugated Oligomers: A Perspective from the Exciton Scattering Approach. J. Phys. Chem. Lett. 2010, 1, 3396-3400.

[35] Li, H., Malinin, S. V., Tretiak, S., and Chernyak, V. Y. Exciton Scattering Approach for Branched Conjugated Molecules and Complexes. IV. Transition Dipoles and
Optical Spectra. J. Chem. Phys. 2010, 132, 124103.

[36] Li, H., Chernyak, V. Y., and Tretiak, S. Natural atomic orbital representation for optical spectra calculations in the exciton scattering approach. J. Phys. Chem. Lett. 2012, 3, 3734-3739.

[37] Cramer, C. J. Essentials of Computational Chemistry; Wiley: West Sussex, England, 2002.

[38] Berresheim, A. J., Muller, M., and Mullen, K. Polyphenylene nanostructures. Chem. Rev. 1999, 99(7), 1747-1786.

[39] Percec, V., Glodde, M., Bera, T. K., Miura, Y., Shiyanovskaya, I., Singer, K. D., Balagurusamy, V. S. K., Heiney, P. A., Schnell, I., Rapp, A., Spiess, H. W., Hudson, S. D., and Duan, H. Self-organization of supramolecular helical dendrimers into complex electronic materials. Nature 2002, 419, $384-387$.

[40] Spitler, E. L., Johnson, C. A., and Haley, M. M. Renaissance of Annulene Chemistry. Chem. Rev. 2006, 106, 5344-5386.

[41] Yanai, T., Tew, D. P., and Handy, N. C. A new hybrid exchange-correlation functional using the Coulombattenuating method (CAM-B3LYP). Chem. Phys. Lett. 2004, 393, 51-57.

[42] Limacher, P. A., Mikkelsen, K. V., and Lüthi, H. P. On the Accurate Calculation of Polarizabilities and Second Hyperpolarizabilities of Polyacetylene Oligomer Chains Using the CAM-B3LYP Density Functional. J. Chem. Phys. 2009, 130, 194114.

[43] Frisch, M. J. et al. Gaussian 09 Revision A.01. Gaussian Inc. Wallingford CT 2009.

[44] Löwdin, P. O. On the Non-Orthogonality Problem Connected with the Use of Atomic Wave Functions in the Theory of Molecules and Crystals. J. Chem Phys. 1950, 18, 365-375.

[45] Reed, A. E., Curtiss, L. A., and Weinhold, F. Intermolecular Interactions From A Natural Bond Orbital ; DonorAcceptor Viewpoint. Chem. Rev. 1988, 88, 899-926.

[46] Glendening, E. D., Reed, A. E., Carpenter, J. E., and Weinhold, F. NBO Version 3.1.

[47] Li, H., Malinin, S. V., Tretiak, S., and Chernyak, V. Y. Effective tight-binding models for excitons in branched conjugated molecules. J. Chem. Phys. 2013, 139, 064109.

[48] Catanzaro, M. J., Shi, T., Tretiak, S., and Chernyak, V. Y. Counting the Number of Excited States in Organic Semiconductors Systems Using Topology. J. Chem. Phys. 2015, 142, 084113.

[49] Li, H., Catanzaro, M. J., Tretiak, S., and Chernyak, V. Y. Excited-State Structure Modifications Due to Molecular Substituents and Exciton Scattering in Conjugated Molecules. J. Phys. Chem. Lett. 2014, 5, 641-647.

[50] Shi, T, Li, H., Tretiak, S., and Chernyak, V. Y. How Geometric Distortions Scatter Electronic Excitations in Conjugated Molecules. J. Phys. Chem. Lett. 2014, 5, 39463952. 
FIG. 1: Illustration of the ES approach. (a) structure of a conjugated molecule; (b) exciton scattering picture on the quasi-1D graph of the molecule; (c) building blocks of phenylacetylene (PA) molecules; (d) linear PA molecule consists of 10 repeat units; (e) exciton-scattering patterns given by the contour plots of transition density matrices from ground state to excited state in the molecule shown in (d).

FIG. 2: (a) Dispersions of the lowest energy exciton band in phenylacetylene (PA) and ladder poly-para-phenylene (PPP) oligomers (whose linear segments are shown in the insets) derived from the TD-DFT calculations. (b) The reflection phases $\phi_{T}$ and $\phi_{X}$ of the unmodified (hydrogen-terminated) and substituted molecular termini. Inset: linear PA molecules substituted by $\mathrm{X}\left(\mathrm{X}=\mathrm{NH}_{2}\right.$ and $\left.\mathrm{NO}_{2}\right)$ on one end used to derive the reflection phases.

FIG. 3: (a) The transition charge $q(\omega)$ and dipole $d(\omega)$ parameters of PA repeat unit. (b) The transition charge parameters $q^{(X)}(\omega)$ of donor $\left(-\mathrm{NH}_{2}\right)$ /acceptor $\left(-\mathrm{NO}_{2}\right)$ substituted and neutral $(-\mathrm{H})$ termini. (c) Same as (b) but the transition dipole parameters $d^{(X)}(\omega)$.

FIG. 4: Comparison of the optical spectra obtained from quantum-chemical (TDDFT) calculation (top panel) and the ES approach (bottom panel) for molecules shown in the inset.

FIG. 5: (a) meta-conjugated $(M)$ and ortho-conjugated $(O)$ $V$-type vertices. The atoms in the region marked with " $(+)$ " should be considered as part of the vertex, whereas those in the region marked with "(-)" should be "deducted" from the vertex due to the overlap of the attached repeat units. The coordinate system for the transition dipole in the $V$ joints is shown on the right. (b) Scattering phases of $M$ and $O$ joints, subscripts 0 and 1 represent the two symmetries (symmetric and antisymmetric modes) of the scattering. (c) The transition charge and dipole parameters of the $M$ joint. (d) Same as (c) but of the $O$ joint.

FIG. 6: (a) The scattering phases of symmetric $Y$-type joint. (b) The transition charge and dipole parameters of $Y$ joint. Insets: The overlapping atoms from the attached repeat units are labeled with "(-)"; the coordinate system for the transition dipole is shown on the right $\left(d_{x}^{Y}=d_{y}^{Y}=d^{Y}\right)$. (c) Comparison of the absorption spectra obtained from TD-DFT calculations (top panel) and the ES approach (bottom panel) for dendrimer shown in the inset. 

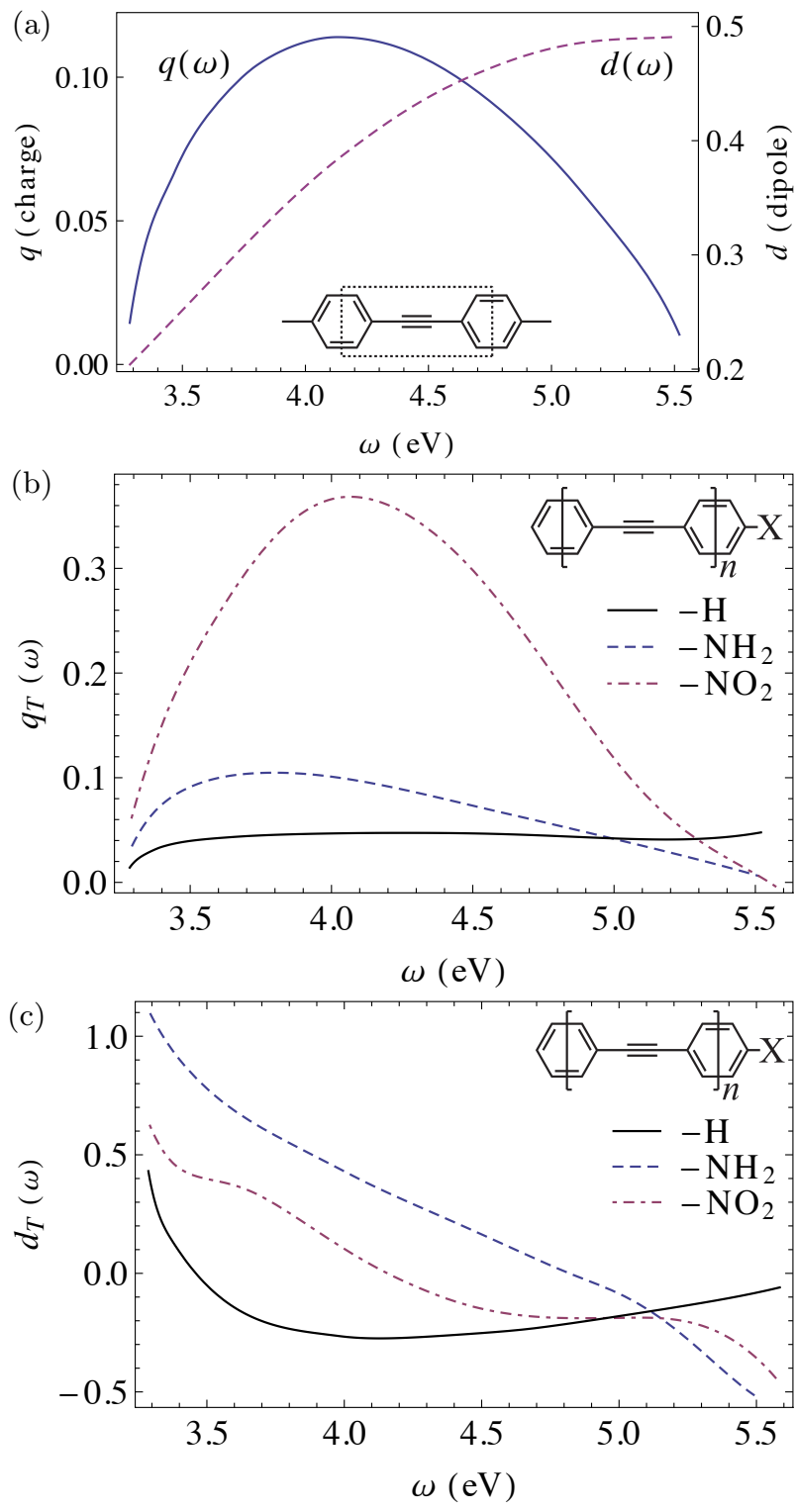

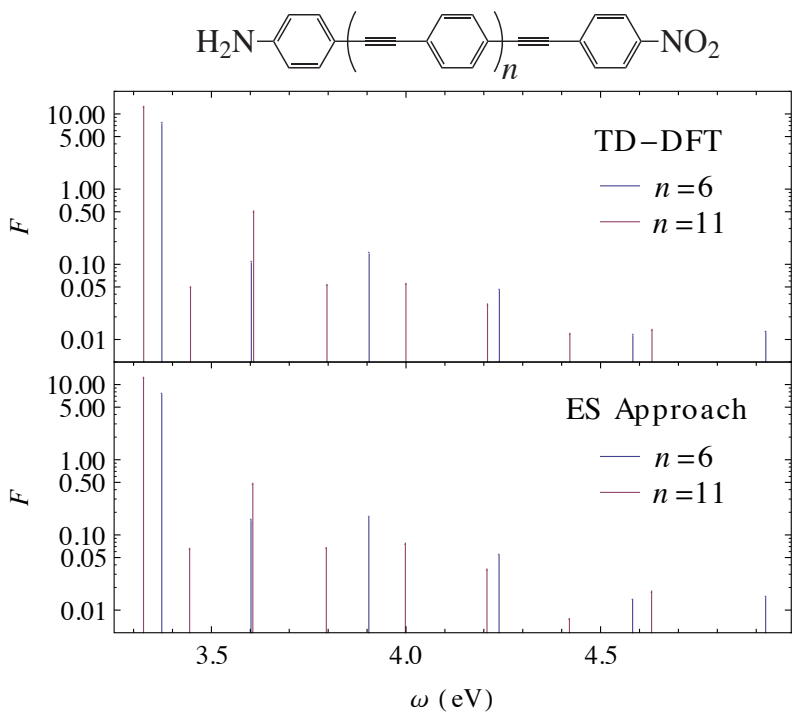
(a)
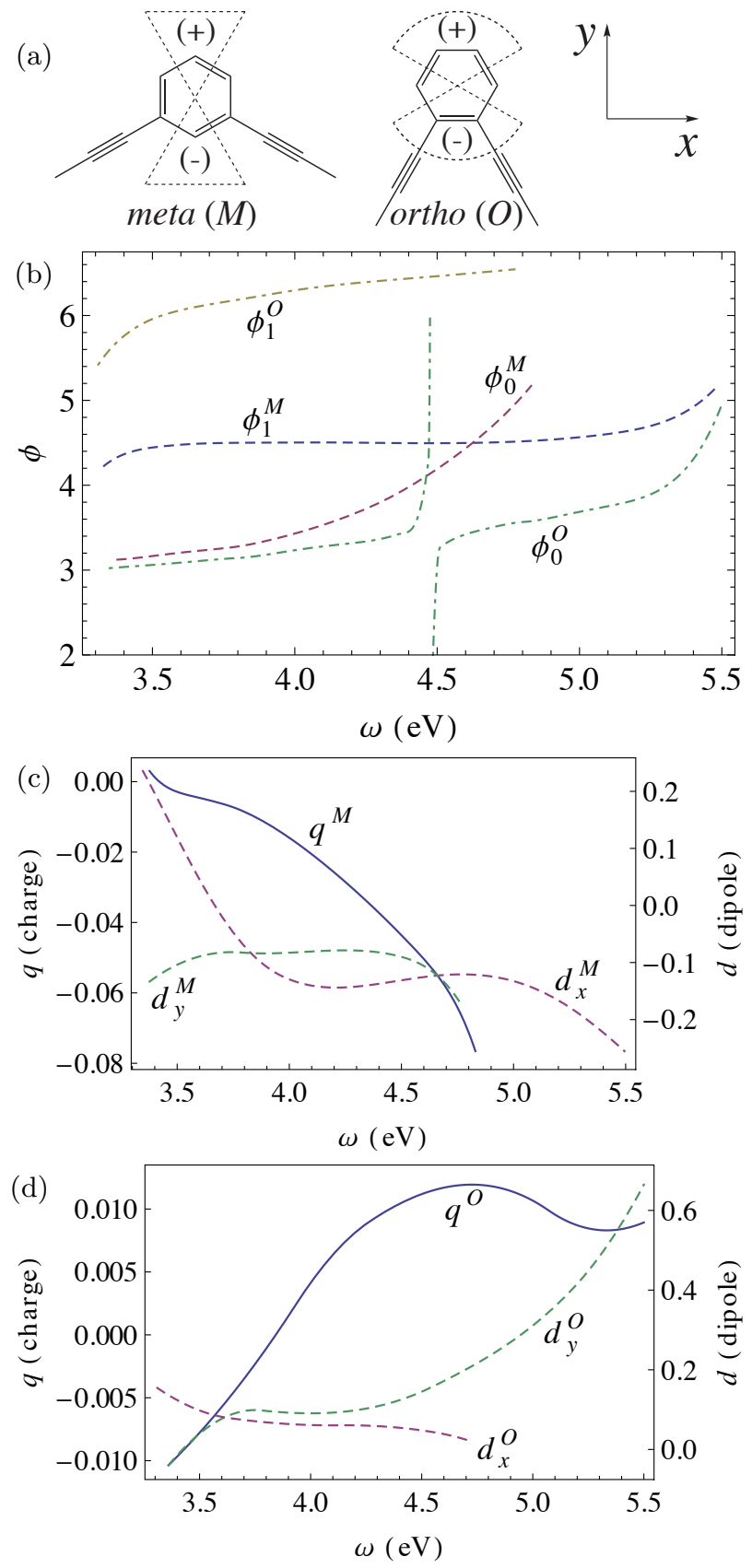


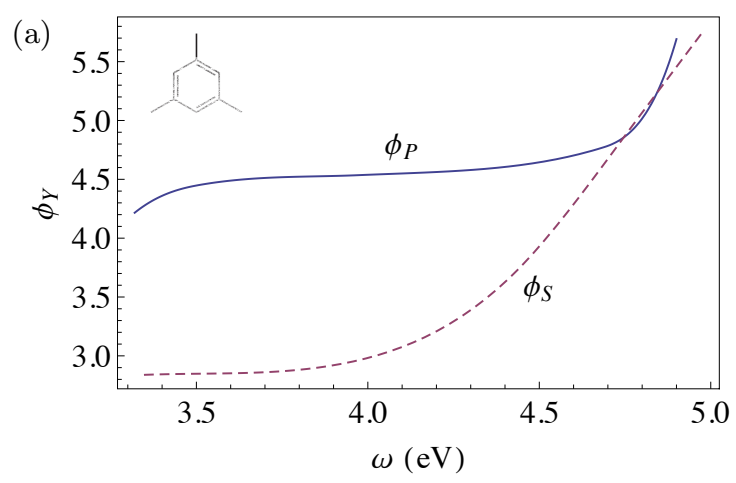

(b)
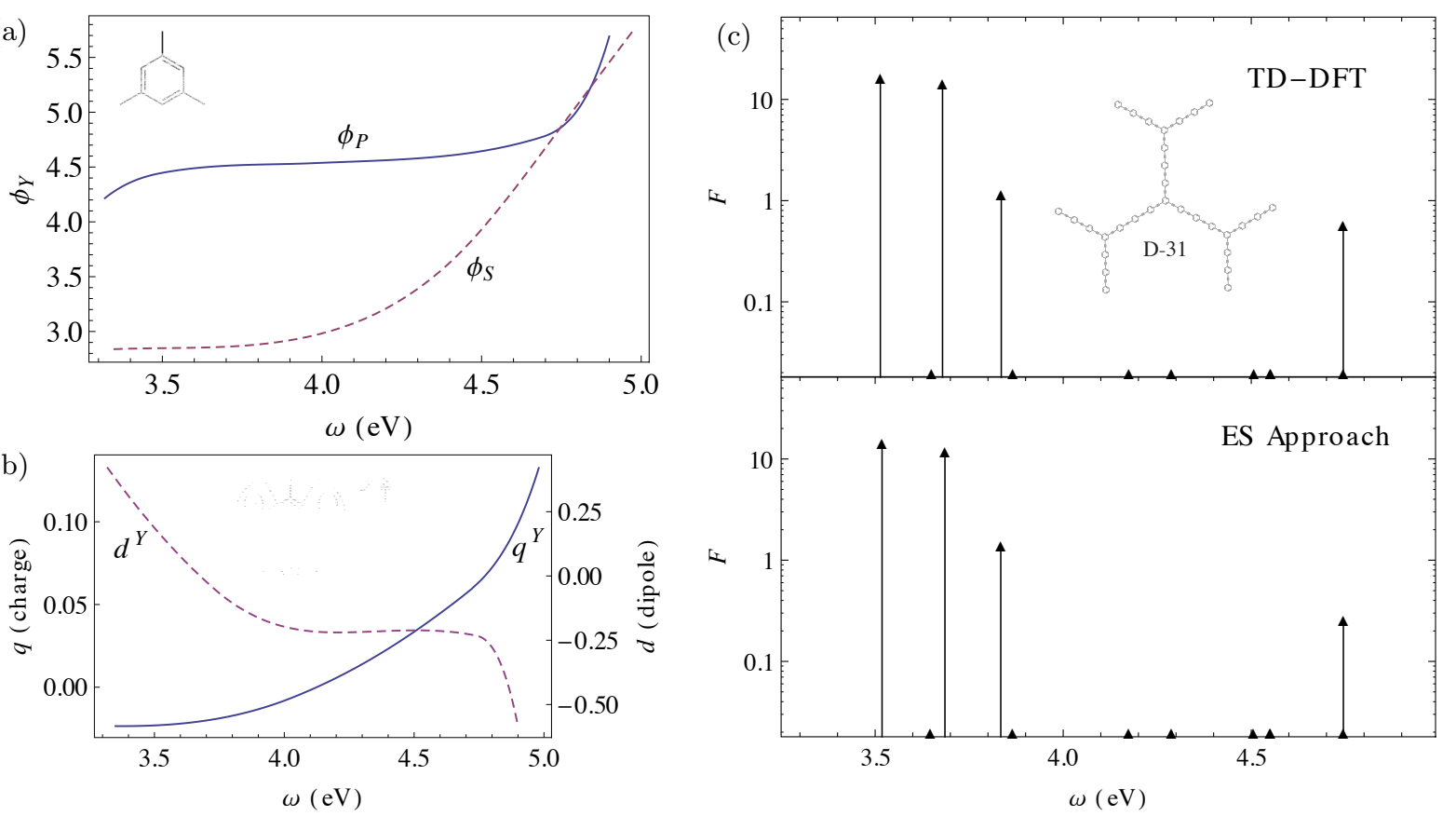


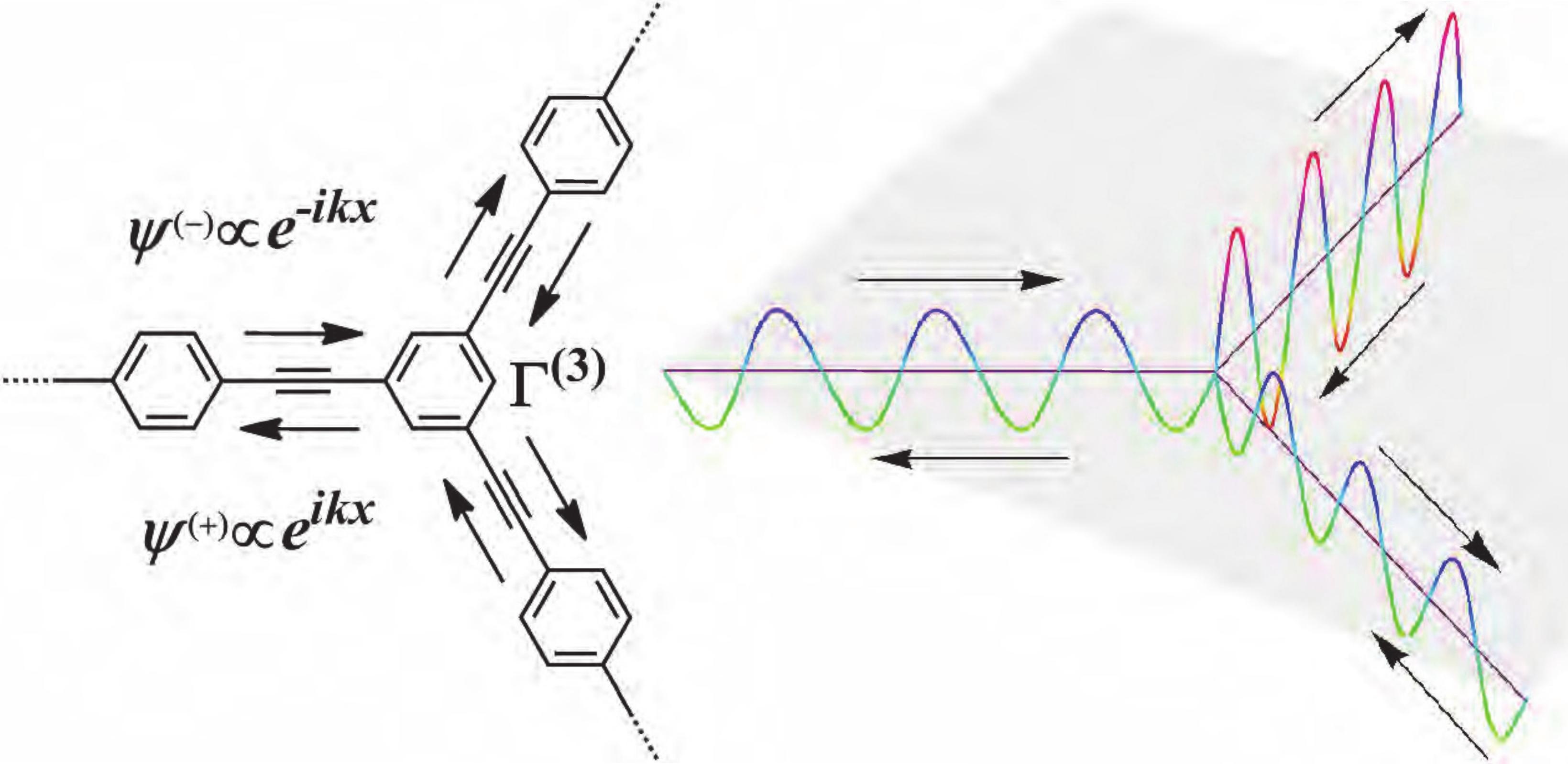

